\title{
A GIS ANCHORED TECHNIQUE TO OBTAIN OPTIMAL PATH THROUGH MINIMAL ALTERATION OF LAND TYPE
}

\author{
Anirban Chakraborty ${ }^{1}$, J. K. Mandal ${ }^{2}$, T. Neogi $^{3}$ \& S. Nayek ${ }^{3}$ \\ ${ }^{1}$ Department of Computer Science \\ Barrackpore Rastraguru Surendranath College \\ Barrackpore, Kolkata-700 120, W.B., India \\ theanirbandrediffmail.com \\ ${ }^{2}$ Department of Computer Science \& Engineering University of Kalyani \\ Kalyani, Nadia, W.B., India \\ jkm.cse@gmail.com \\ ${ }^{3}$ M. Sc. Students, Department of Computer Science \\ Barrackpore Rastraguru Surendranath College \\ Barrackpore, Kolkata-700 120, W.B., India \\ greenvalley 74 @yahoo.com, subhojitnayek007@yahoo.co.in
}

\begin{abstract}
This paper presented a technique to organize the digitized map of a very large area, using a flat-file system (without using databases); depicting necessary and useful information regarding land usage of the area, which can also be viewed as a land-type-data bank, where all the necessary information regarding the type of the land, owner etc. are kept. While associating data with each individual region, a priority value is also incorporated, where a lower value of priority signifies, higher possibility of the land to be selected for acquisition. As per needs to acquire lands for the construction of new utility services such as railway tracks, roads etc., the technique, upon input the source and the destination location, suggests the most suitable path, in terms of optimal costs, which is dependent on the parameters like compensation needed to pay during land acquisition (compensation amount increases, for acquiring more fertile lands), the length of the path etc. The suggested optimal path is presented onto the map graphically assisting the engineers involved in the work for proper implementation and utilisation of the land.
\end{abstract}

\section{KEYWORDS}

Digitized map, flat-file-systems, land-type-data-bank, priority, land acquisition, optimal cost path.

\section{INTRODUCTION}

Agriculture is the backbone of Indian Civilization and still now the main usage of land in our country is for the purpose of agriculture. But, with the passage of time, not only the population of our country (as well as of the whole world) is increasing by leaps and bounds; buses, cars, rails, as well as other types of communication are increasing day by day to cater to the need of growing 
population. However, building roads and railway tracks in turn require land acquisition, because these constructions must have a large area[1,2]. Although the term "Land acquisition" sounds quite a simple one, but at least in the Indian context, the bygone years are awarded with the mishaps of the hostile atrocities of humanity towards the fellowmen over the land acquisition issue and called for the contentious criticisms and beguiled controversies that carved its way through the daily media, gave rise to many agitations in local and national level[3]. The amount of agitation could be minimized some how, if only the less fertile lands are being selected for acquisition[4,5].

Although it sounds fascinating, but for a very large area under consideration, practically it is perhaps manually impossible to consider all the possible routes one by one, from source to destination, covering the fertile lands as less as possible and then to select the optimal one path, after comparing their costs under few predefined parameters[6,7,8,9,10,11,12,13,14,15,16,17]. The work present proposes to find a remedy for this lingering problem.

Addressing the aforesaid problem, the present work provide the planning for the road to be built in such a way, curved towards the remedy for this lingering problem of land acquisition, by suggesting acquiring lands which are very less fertile along with focusing on some other parameters while choosing a land, which could be altered by an individual for a suitable situation. The proposed technique developed, taking digitized map as input which is a precursor to the system.

Section 2 of this paper deals with the scheme of the techniques. The results of implementations are given in section 3. Analysis and comparisons are outlined in section 4 that of conclusions are drawn in section 5 and references in section 6 .

\section{SCHEME}

The basic objective of the work are visualize using following sub-systems:

$>$ A graphical user interface, by which raster map of a very large area (which is nothing but a pre-stored image) can be converted into a vector map by means of digitization

$>$ Data collected by means of survey, regarding the owner of the land, priorities of the land etc. are associated with the digitized map

$>$ On entering the source and destination regions, the optimal path between these two, causing minimal alteration of land is obtained.

Section 2.1 explains the basic approach with associated data structures needed to fulfil the objectives. Section 2.2 deals with the algorithm used in the proposed technique and finally the construction process of the optimal path through back-tracking is given in section 2.3.

\subsection{Methodology}

Proposed work embedded with a previously map. In context of Indian scenario, it may be a map of the entire Block (under any district), where at the time of digitization, the associated information regarding each tiny lands are given, such as, type of land (some numerical values may be assigned for this purpose, such as 0 for deserted land, 1 for one-crop field and finally a very high values for 
those lands, which can't be acquired, due to having already some constructions like temples, schools, factories etc.), the owner of the lands and so on.

The map of any large region under consideration, consisting of a number of small discrete lands may be viewed as a connected graph, where each small land is regarded as a node. For demonstration purpose, one such area is shown in fig. 1, consisting of 7 regions. Here the user is to feed the information regarding the adjacency of two lands. It is to be noted that two lands will be said to be adjacent only when they have some common boundary. This information can be gathered through user friendly screen (only by mouse clicking) and the information is then stored in an "Adjacency Matrix". In figure 1, the regions which are adjacent to one another, are linked with straight lines (i.e. a connection exists between them).

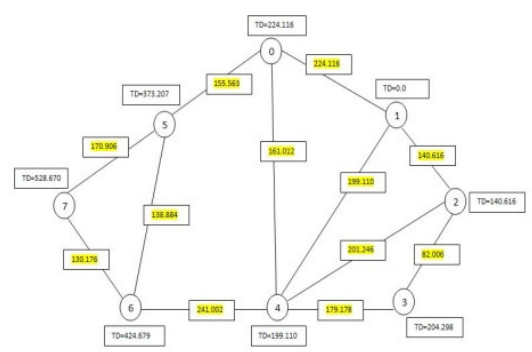

Fig. 1: The graphical representation of the area under consideration

The problem now may be viewed as finding the shortest path from source to destination point, keeping in mind to traverse through the less priority regions, but at the same time the length should not be much more. From architectural point of view some more parameters, like soft lands or curvy lands etc. may also be considered, while choosing lands for acquisition.

In the graph shown in figure 1 , let a path has to construct from node 5 to node 1 . The magnitude mentioned over a node specifies the direct distance (centroid to centroid) between that node and the destination. The magnitudes which are mentioned inside the box over the edge denote the length of that edge i.e. it is the direct distance between the two nodes connected by that edge. Let us consider the priorities of the regions as given in table 1 .

Table 1: Table showing prorities of the regions (corresponding to fig. 1)

\begin{tabular}{|l|c|c|c|c|c|c|c|c|}
\hline Regions & 0 & 1 & 2 & 3 & 4 & 5 & 6 & 7 \\
\hline Priorities & 100 & NIL & NIL & 5 & 6 & NIL & NIL & NIL \\
\hline
\end{tabular}

Here, a NIL under priority means, that the user has not entered it explicitly, so a 0 (zero) value is assigned automatically. To make any region forbidden (which much not be acquired anyhow), a very high priority value is assigned with it.

For feeding the adjacencies or while input the start and destination region; only clicking inside the regions are sufficient. Using the Ray-Casting algorithm, it is determined that inside which polygon (region) the clicked point belongs. 


\subsection{Proposed Algorithm}

Two arrays OpenSet and CloseSet are used for the purpose of keeping track of the visited regions and the distance between source and any other region is measured by cumulatively adding the distances between all the intermediate regions travelled so far to reach at the present region, while the distance from that region to the destination region is just the centroid to centroid distance between the two.

The main motivation behind the present technique is to select only the low priority regions for construction of the path, while keeping in mind not to make the length very much. To make a balance between these two, while calculating the value of cost factor of selecting any particular region, a weightage factor of 0.75 is associated with the priority value and a factor of 0.25 is associated with the distance traversed. This two values has been adopted after performing a small survey of the situation in West Bengal, however this weightage factor will obviously get changed with time and place and has to be determined by proper survey. Although for the sake of simplicity, only two parameters are considered for the present work, but many more parameters like softness of the land, its inclination value etc. can easily be considered as attributes. So based on the architectural needs, parameters may be adopted within the algorithm. The step-wise procedure of the technique is as follows

Step I: Initially the region ID of the REGION, as input by user, is only placed in OpenSet. Each time, for any region, two values are being considered, one is g value(say), which signify the distance traversed till this intermediate region is reached while traversing from the source and $\mathrm{h}$ (say), is the Euclidian distance from this intermediate point to the destination one. The required functional value $f$ is the $25 \%$ of summation of these two (i.e. $f(x)=g(x)+h(x)$ ) plus $75 \%$ obtained from priority of the region. Thus initially for the source region, its $\mathrm{g}$ value is set to 0 and its $\mathrm{h}$ value as obtained from graph. Initially, CloseSet is made empty.

Step II: Until a goal node is found, the following procedure is repeated:

If there are no region ID on OpenSet, failure is reported. Otherwise, the ID on OpenSet with the lowest calculated $\mathrm{f}$ value is picked, designated as BESTNODE. It is removed from OpenSet and is placed on ClosedSet. If BESTNODE is a goal node, then the procedure halts and a solution is reported. Otherwise, all the regions which are adjacent to the BESTNODE (may be designated as SUCCESSOR), are placed at OpenSet. For each SUCCESSOR, the following procedure is adopted:

a) The path from SUCCESSOR pointing back to BESTNODE is memorised. This backwards link will make it possible to recover the path once a solution is found (section II.C).

b) If SUCCESSOR is the same as any region on OpenSet (i.e., it has already been generated but not processed) then it is designated as OLD. Since this node already exists in the graph, one can throw SUCCESSOR away and add OLD to the list of BESTNODE's successors. Now it has to decide whether OLD's parent link should be reset to point to BESTNODE. It should be, if the path just found to SUCCESSOR is cheaper than the current best path to OLD (since SUCCESSOR and OLD is really the same node). So it has to examine whether it is cheaper to get to OLD via its current parent or to SUCCESSOR via BESTNODE by comparing their $f$ values. If $f(O L D)$ is cheaper (or just as cheap), then nothing is done. If 
$\mathrm{f}$ (SUCCESOR) is cheaper, then OLD's parent link is reset to point to BESTNODE and $\mathrm{f}$ (SUCCESSOR) is recorded.

c) If the SUCCESSOR is the same as any region on ClosedSet (i.e., it has already been generated and processed), then this SUCCESSOR is skipped and the next node with least $\mathrm{f}$ value is picked and the previous steps are followed.

d) If the SUCCESSOR was not already on either OpenSet or ClosedSet, then it is put on OpenSet, $\mathrm{f}$ (SUCCESSOR) is computed using the procedure mentioned in Step I and Step II is repeated.

\subsection{Construction of Optimal path}

On finding the destined region, a backtracking mechanism is incorporated, to point out the path graphically on the map (digitized). Since the target region is in the ClosedSet (a condition for successful halt of the procedure), inspection starts from that region and its immediate parent is found. A line is drawn connecting the two regions (a line joining the centroids of these two). From this parent region, again its immediate region is found and the path connecting these two is constructed. The method is repeated till the source node is reached and the path reaching this node is drawn. Obviously at this point the complete optimal path, meeting the objective of this work is obtained and shown graphically onto the map.

\section{Implementation \& Results}

The results of implementation and its operations have been done using Net Beans (Java), which is based on file systems without using databases. The work starts with choosing a map from any location of the computer, digitizing it (fig $2 \mathrm{a}$ and $2 \mathrm{~b}$ ) and then feeding data associated with various regions (fig $2 \mathrm{c}$ ).

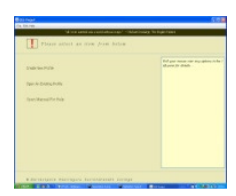

Fig. 2(a)

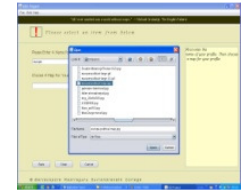

Fig. 2(b)

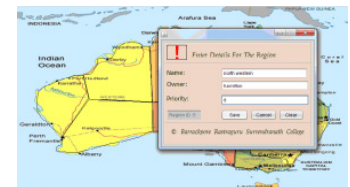

Fig 2(c)

Fig. 2: Digitization \& Data Association

The next step is to feed the "Adjacency" information, where the information is saved by clicking "Accept" button and upon clicking "Show Adj." button, two adjacent regions are shown by a connected red line (fig $3 a$ and $3 b$ ).

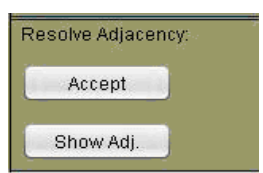

Fig. 3(a)

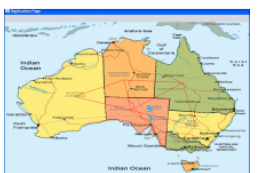

Fig. 3(b)

Fig. 3: Incorporating and displaying adjacency information 
Finally the source and the destination points are given by clicking "Give Points" button, after which the best suggested path between them is generated and displayed graphically upon clicking onto the "Generate" button (fig $4 \mathrm{a}$ and $4 \mathrm{~b}$ ).

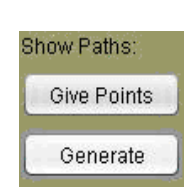

Fig. 4(a)

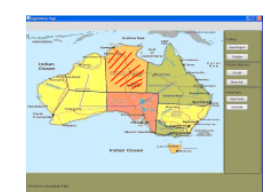

Fig. 4(b)

Fig. 4: Optimal path between source \& destination

\section{ANALYSIS \& COMPARISONS}

In the proposed technique, a path of the lowest cost is followed, keeping a sorted priority queue of alternate path segments along the way. If, at any point, a segment of the path being traversed has a higher cost than another encountered path segment, it abandons the higher-cost path segment and traverses the lower-cost path segment instead. This process continues until the goal is reached.[4]

Compared to all informed search algorithms, the proposed technique also searches the routes which appeared to be most likely to move forward towards the goal, but the advantage is that it also takes the distance already travelled into account. The function of the same can be expressed as $f(x)=g(x)+h(x)$, where the $g(x)$ part of the heuristic is the cost from the starting point, not simply the local cost from the previously expanded node.

The advantages of this proposed technique are as follows:

* During digitizing, both the mouse move and click can be adopted simultaneously, without specifying the technique beforehand, which makes the process less timeconsuming and user-friendly.

* Editing is possible to accommodate variable needs, for example, here only the priority and the length of the path has been considered, but if one desires, other aspect like curviness of the land, softness/hardness of the soil etc. may also be taken into account.

* It is based on purely flat file systems, not dependent on any particular operating systems and DBMS. Instead, if databases are used then, not only the cost would be increased, the portability will also be lost, as most of the databases are proprietary.

* The graphical trace of the proposed roadway is displayed, from which the idea about the lands which are needed to acquire is obtained. Moreover, from the data associated with each land the information regarding the owner and other necessary information could be obtained.

An automated system solely mean for directing land acquisition, as like the present work is not available, but some of the GIS based tool can be tiled to do these tasks only incorporating some modifications. For example, using TNTMips, although digitization and data association is possible, but it will not help to find the optimal cost path, on the basis of the priorities of the acquired land, until and unless some modifications are done. 


\section{CONCLUSiOnS}

Agriculture is the backbone of the country, but to serve better to the huge population of this country and for accepting the challenge of improvement of the communication system, new roadways/ railway tracks are to construct, which in turn require land acquisition, leading to many agitations in regional/national level. This work is an attempt, which suggests constructing a roadway from one point to another by acquiring less fertile lands as much as possible, hoping to minimize the people (farmers) agitations at least up to a certain extent.

\section{ACKNOWLEDGMENT}

The authors express a deep sense of gratitude to the Department of Computer Science, Barrackpore Rastraguru Surendranath College, Kolkata-700 120, India and Department of Computer Science and Engineering, University of Kalyani for providing necessary infrastructural support for the work and to the UGC for financial support under Minor Research Project scheme (sanction number F. PSW-180/11-12 ERO, dated 25.01.2012).

\section{REFERENCES}

[1] Anirban Chakraborty, J. K. Mandal, Arun Kumar Chakraborti, A File base GIS Anchored Information Retrieval Scheme (FBGISIRS) through Vectorization of Raster Map, International Journal of advanced research in computer science, ISSN No. 0976-5697 volume-2 No.4, pp 132-138, July August, 2011 .

[2] Anirban Chakraborty, J. K. Mandal, N. Banerjee, P. Patra, A GIS based Interlinked Information Retrieval of a Large Database using KD Tree, Conference Proceedings "UGC-Sponsored National Symposium on Emerging Trends in Computer Science(ETCS 2012)", ISBN number 978-81-9218082-3, , pp 84-89, 20-21 January 2012

[3] Elaine Rich, Kevin Knight, Artificial Intelligence, Tata McGraw Hill, Seventh Reprint 2011.

[4] http://en.wikipedia.org/wiki/A*_search_algorithm, date of access 20 May, 2012.

[5] http://www.cs.trincoll.edu/ ram/cpsc352/notes/astar.html, date of access, 24 April,2012.

[6] http://theory.stanford.edu/ amitp/GameProgramming/AStarComparison.html, accessed in 15 May,2012.

[7] http://www.policyalmanac.org/games/aStarTutorial.htm, date of access, 18 May,2012.

[8] http://en.wikipedia.org/wiki/Geographic_information_system, date of access 24 February, 2012.

[9] http://www.gis.com/content/what-gis, date of access 24 February, 2012.

[10] http://www.gisindia.in, date of access, 22 March, 2012.

[11] http://www.esriindia.com, date of access, 28 March,2012.

[12] http://www.egsc.usgs.gov/isb/pubs/gis_poster, date of access, 19 April, 2012

[13] http://www.mapsofindia.com/gis-services.html, date of access, 23 March, 2012.

[14] http://www.gismaps.in, date of access, 27 February, 2012

[15] http://www.maptell.com, date of access, 25 February,2012

[16] http://www.docs.oracle.com/javase/tutorial, date of access, 12 May, 2012

[17] http://www.tutorialspoint.com/java/index.htm, date of access, 14 May, 2012 